\title{
Fluid motion recovery by coupling dense and parametric vector fields
}

\author{
Étienne Mémin and Patrick Pérez \\ IRISA/INRIA \\ Campus de Beaulieu, 35042 Rennes Cedex, France \\ memin@irisa.fr, perez@irisa.fr
}

\begin{abstract}
In this paper we address the problem of estimating and analyzing the motion in image sequences that involve fluid phenomena. In this context standard motion estimation techniques are not well adapted and more dedicated approaches have to be designed. In this prospect, we propose to estimate in a joint and cooperative way a dense motion field and a peculiar parametric representation of the flow. The parametric model issues from an extension of Rankine vortex model and includes a laminar flow field. Dense and parametric fields are estimated by minimizing a robust global objective function thanks to a specific alternate scheme. The method has been validated on different kinds of meteorological image sequences.
\end{abstract}

\section{Background: Fluid Motion Estimation}

In a number of domains, image sequences that involve fluid phenomena, have to be analyzed: In environmental sciences (oceanography, meteorology, climatology, etc.), ocean and atmosphere evolutions are observed via satellite sensors [5, 9]; In medical imaging, blood flow can be monitored by angiography [14]; In the field of fluid mechanics, aero- and hydro-dynamics experiments now routinely produce lots of video data $[7,10,15]$. In all these domains of applications, camera offers in a versatile and non-intrusive way, huge amounts of spatio-temporal data, as opposed to in situ measurement techniques that are often complex, very specific, intrusive, and that only provide with sparse data. With these latter techniques, however, sought quantities are directly measured with dedicated probes, whereas, within image sequences, the relevant information has to be extracted from the luminance data.

The analysis of motion in such sequences is particularly challenging due to the great deal of spatial and temporal distortions that luminance patterns exhibit in imaged fluid phe- nomena. Standard techniques from Computer Vision, originally designed for quasi-rigid motions with stable salient features, are not well adapted in this context. The design of alternate approaches dedicated to fluid motion thus constitutes a widely open domain of research. Our work is a contribution in this direction.

As in standard motion analysis, two types of motion information can be sought. First, dense velocity (or displacement) fields [5,9] constitute precious sources of information which can serve either as validation basis, or as input data for numerical models (e.g., in short-term weather prediction). They are also used for visualization purposes, and allow to compute other quantities of interest, such as the vorticity of the flow [15]. Second, some salient structures may be sought. Vortices $[14,16]$, and more generally singular points $[5,7,10]$ are kinematic entities of particular interest: they provide a compact and relevant representation of fluid flows [7], they retain key information for the understanding of phenomena (e.g., vortices in study of turbulence [15], depressions in meteorology [9]), and they provide tokens for tracking purposes [10]. Such entities can be extracted $a$ posteriori from estimated velocity fields $[5,7,14,16]$. They can also be recovered directly from images $[7,10]$.

We suggest here that both types of information should be extracted in a joint and cooperative way. To this end we introduce, on the basis of a joint estimation segmentation approach [12], a coupled approach which mixes the optical flow technique proposed in [13] with an original non-linear parametric flow modeling based on vortices, sources and sinks.

\section{Dense/Parametric Robust Modeling}

Dense motion estimation aims at estimating a velocity map $\boldsymbol{w}=\left\{\boldsymbol{w}_{s}, s \in S\right\}$ at each point of the rectangular pixel lattice $S$, based on brightness function $f(t)=\{f(s, t), s \in$ $S\}$ at two consecutive instants $t$ and $t+1$. Assuming temporal constancy of the brightness function, standard optic-flow 
estimation rely on a differential equation known as opticflow constraint equation (OFCE):

$$
\nabla f(s, t)^{T} \boldsymbol{w}_{s}+f_{t}(s)=0
$$

where $\nabla f$ stands for the spatial gradient of $f$ and $f_{t}\left(s, \boldsymbol{w}_{s}\right) \triangleq f(s, t+1)-f(s, t)$ denotes the finite difference approximation of the temporal derivative. This equation issues from a linearization of the brightness constancy assumption. It may also be seen as the material derivative of $f$ (i.e., the rate of change of $f$ as observed when moving with point $s$ ).

The OFCE being known to be not valid in general for large displacements (the linearity domain of the luminance function is drastically reduced for long range displacements, as well as at sharp edge locations) an incremental version of this equation is usually considered. This technique which may be related to non-linear least squares Gauss-Newton method $[1,11]$ is generally used in combination with a standard multiresolution setup [2,6]. In the following, we shall assume to work at a given resolution of such a pyramidal structure. However, one has to keep in mind that the expressions and computations are meant to be reproduced at each resolution level according to a coarseto-fine strategy.

Let us now assume that a rough estimate $\boldsymbol{w}=\left\{\boldsymbol{w}_{s}, s \in\right.$ $S\}$ of the unknown velocity field is available (e.g., from an estimation at lower resolution or from a previous estimation). Based on a linearization of the constancy brightness assumption from time $t$ to $t+1$ around $\boldsymbol{w}$, a small increment field $\mathrm{d} \boldsymbol{w} \in \Omega \subset(\mathbb{R} \times \mathbb{R})^{S}$ can be estimated as:

$$
\arg \min _{\mathrm{d} \boldsymbol{w}} H_{1}(\mathrm{~d} \boldsymbol{w} ; f, \boldsymbol{w})+\alpha H_{2}(\mathrm{~d} \boldsymbol{w} ; \boldsymbol{w}),
$$

with $[2,11]$ :

$$
\begin{aligned}
& H_{1} \triangleq \sum_{s \in S} \rho_{1}\left[\nabla f\left(s+\boldsymbol{w}_{s}, t+1\right)^{T} \mathrm{~d} \boldsymbol{w}_{s}+f_{t}\left(s, \boldsymbol{w}_{s}\right)\right], \\
& H_{2} \triangleq \sum_{<s, r>\in \mathcal{C}} \rho_{2}\left[\left\|\left(\boldsymbol{w}_{s}+\mathrm{d} \boldsymbol{w}_{s}\right)-\left(\boldsymbol{w}_{r}+\mathrm{d} \boldsymbol{w}_{r}\right)\right\|\right],
\end{aligned}
$$

where $\alpha>0, \mathcal{C}$ is the set of neighboring site pairs lying on grid $S$ equipped with some neighborhood system $\nu$, $f_{t}\left(s, \boldsymbol{w}_{s}\right) \triangleq f\left(s+\boldsymbol{w}_{s}, t+1\right)-f(s, t)$ is now the displaced frame difference, and $\rho_{1}$ and $\rho_{2}$ are standard robust $M$ estimators (with hyper-parameters $\sigma_{1}$ and $\sigma_{2}$ ). Such functions penalize the deviations both from the data model (i.e., the OFCE) and from the first-order smoothing prior.

The dense estimator (1-3) is general; it is only based on the assumptions of luminance conservation (first term) and of spatial smoothness of the velocity (second term). It does not rely on any prior knowledge about typical fluid flows.

In most situations, it is relevant to consider that fluid motion is composed of three parts: a smooth laminar component, a divergence-free component stemming from a few
Figure 1. Example of vortex $\left(\operatorname{rot}_{i}>0\right)$, source $\left(\operatorname{div}_{i}>\right.$ $0)$, and translated swirl with shear $\left(a_{i}, b_{i}, \operatorname{rot}_{i}, \operatorname{div}_{i}\right.$ and shear $\left._{i} \triangleq \sqrt{\left(c_{i}-f_{i}\right)^{2}+\left(e_{i}+d_{i}\right)^{2}}>0\right)$.

vortices, and an irrotational component produced by a few sinks/sources. Vortices correspond to localized concentrations of vorticity $\operatorname{rot} w \triangleq u_{x}-u_{y}$, whereas sinks and sources are associated to analog concentrations of divergence $\operatorname{div} \boldsymbol{w} \triangleq u_{x}+v_{y}$.

Extending Rankine vortex model [14], we introduce an original unified modeling of these entities. Let a vortex/sink/source be located at $s_{i}=\left(x_{i}, y_{i}\right)$. In a certain neighborhood of $s_{i}$, the velocity field is approximated by a linear model. Beyond this neighborhood, the same linear expression is kept, but scaled by the inverse of the squared distance to $s_{i}$. Assuming a circular neighborhood $D_{i}$ of radius $r_{i}$ around $s_{i}$, we thus consider the following parametric velocity field:

$\boldsymbol{w}_{i}(s) \triangleq \min \left(1, \frac{r_{i}^{2}}{\left\|s-s_{i}\right\|^{2}}\right) \cdot\left(\left[\begin{array}{l}a_{i} \\ b_{i}\end{array}\right]+\left[\begin{array}{ll}c_{i} & d_{i} \\ e_{i} & f_{i}\end{array}\right]\left[\begin{array}{l}x-x_{i} \\ y-y_{i}\end{array}\right]\right)$,
for $s=(x, y) \in S$.

One can verify that the divergence and the vorticity of this field decrease as $\left\|s-s_{i}\right\|^{-2}$ beyond $D_{i}$, and they respectively amount to $\operatorname{div}_{i} \triangleq c_{i}+f_{i}$ and $\operatorname{rot}_{i} \triangleq e_{i}-d_{i}$ within this disk. Vortices correspond to significantly non-zero values of $\operatorname{rot}_{i}$. Significant positive (resp. negative) values of $\operatorname{div}_{i}$ correspond to sources (resp. sinks). Both situations can be combined within swirls. See examples in Figure 1.

If $K$ vortices/sinks/sources are present, the total field results from the sum of all $\boldsymbol{w}_{i}$ 's with some laminar flow which "transports" them. We make interact these different modeling ingredients with the dense field through a robust goodness-of-fit cost function:

$$
\begin{aligned}
& H_{3}\left(\mathrm{~d} \boldsymbol{w}, \boldsymbol{w}_{\text {lam }}, \theta_{1} \cdots \theta_{K} ; \boldsymbol{w}\right) \triangleq \\
& \quad \sum_{s} \rho_{3}\left[\left\|\boldsymbol{w}_{s}+\mathrm{d} \boldsymbol{w}_{s}-\left(\boldsymbol{w}_{\text {lam }}(s)+\sum_{i=1}^{K} \boldsymbol{w}_{i}(s)\right)\right\|\right]
\end{aligned}
$$

where $\boldsymbol{w}_{\text {lam }}$ denotes the laminar part of the flow and $\theta_{i} \triangleq$ $\left(s_{i}, r_{i}, a_{i}, b_{i}, c_{i}, d_{i}, e_{i}, f_{i}\right)^{T}$ gathers the parameters relative to the $i$ th vortex/sink/source. 


\section{Joint Estimation}

Due to the classical choice of non-convex robust functions, the overall energy function may be highly nonconvex. Appropriated minimization schemes must be designed to solve this problem. According to a reformulation of the $M$-estimator, the minimization may be easily handled in an alternate way.

Assuming certain simple conditions (mainly concavity of $\phi(v) \triangleq \rho(\sqrt{v})$, see $[3,4,8]$ for a complete account), any multidimensional minimization problem of the form "find $\arg \min _{x} \sum_{i} \rho\left(g_{i}(x)\right)$ " can be turned into a "semi"-quadratic minimization problem "find $\arg \min _{x, z} \sum_{i}\left[\tau z_{i} g_{i}(x)^{2}+\psi\left(z_{i}\right)\right]$ " involving auxiliary variables (or weights) $z_{i}$ 's continuously lying in $(0,1]$. $\psi$ is an increasing function, depending on $\rho$, and $\tau \triangleq$ $\lim _{v \rightarrow 0+} \phi^{\prime}(v)$. Function $\psi$ may be easily computed from $\rho$ but it is never used in practice. The new minimization is usually led alternatively with respect to $x$ and to the $z_{i}$ 's. The expression being quadratic w.r.t. $x$ if the $g_{i}$ 's are linear, the corresponding minimization is conducted through a standard weighted least squares minimization. In turn $x$ being frozen, the best weights are given by the following closed form $[4,8]$ :

$$
\hat{z}_{i}(x)=\frac{\rho^{\prime}\left[g_{i}(x)\right]}{2 \tau x}=\frac{1}{\tau} \phi^{\prime}\left[g_{i}(x)^{2}\right] .
$$

The overall alternate procedure constitutes an iteratively reweighted least squares estimation.

Applying, these reformulations to our energy function $H_{1}+\alpha H_{2}+\gamma H_{3}$ (with $\gamma$ being some positive parameter) leads to the new global energy function $\mathcal{H} \triangleq \mathcal{H}_{1}+\alpha \mathcal{H}_{2}+$ $\gamma \mathcal{H}_{3}$ where:

$$
\begin{aligned}
& \mathcal{H}_{1} \triangleq \sum_{s \in S} \delta_{s}\left[\boldsymbol{\nabla} f\left(s+\boldsymbol{w}_{s}, t+1\right)^{T} \mathrm{~d} \boldsymbol{w}_{s}+f_{t}\left(s, \boldsymbol{w}_{s}\right)\right]^{2}+\psi_{1}\left(\delta_{s}\right) \\
& \mathcal{H}_{2} \triangleq \sum_{<s, r>\in \mathcal{C}} \beta_{s r}\left\|\left(\boldsymbol{w}_{s}+\mathrm{d} \boldsymbol{w}_{s}\right)-\left(\boldsymbol{w}_{r}+\mathrm{d} \boldsymbol{w}_{r}\right)\right\|^{2}+\psi_{2}\left(\beta_{s r}\right) \\
& \mathcal{H}_{3} \triangleq \sum_{s \in S} \xi_{s}\left\|\boldsymbol{w}_{s}+\mathrm{d} \boldsymbol{w}_{s}-\left(\boldsymbol{w}_{l a m}(s)+\sum_{i=1}^{K} \boldsymbol{w}_{i}(s)\right)\right\|^{2}+\psi_{3}\left(\xi_{s}\right)
\end{aligned}
$$

The appearing auxiliary variables $\delta_{s}$ 's and $\xi_{s}$ 's ly within $(0,1]$. They are attached to the image grid and they respectively account for the data model violations and for the "likeness" degree of the estimated dense field to its parametric representation. The other variables $\beta_{s r}$ 's are attached to the dual edge grid; they capture the spatial discontinuities of velocity between adjacent sites.

Assuming that $\boldsymbol{w}$ and $\boldsymbol{w}_{l a m}$ are available in some way, the estimation of $\mathrm{d} w, K, \theta_{1} \cdots \theta_{K}$, and the different weights is conducted in an alternate way. For a fixed increment field and parametric representation, the minimization of $\mathcal{H}$ with respect to the auxiliary variables is explicitly given by equation (6).

In turn, considering given weights and parametric representation of the velocity field, the minimization with respect to $\mathrm{d} w$ amounts to solving a standard weighted least squares problem. This is conducted here by extending an efficient multi-parametric adaptive multigrid technique introduced in [13].

As for the parametric model estimation, we have to estimate the number of sink/sources/vortices, the size of the disks supporting the linear models of the flow and the affine model assigned to each of them. For a given dense field from which laminar component is removed $\boldsymbol{w}+\mathrm{d} \boldsymbol{w}-$ $\boldsymbol{w}_{\text {lam }}=(u, v)^{T}$, sinks, sources and vortices should be singular points which can be extracted in different ways. As in [5], we use winding numbers. The winding number (or index) of a closed curve in a vector field amounts to the number of turns, $\frac{1}{2 \pi} \oint d\left(\tan ^{-1} u / v\right)$, that the field undergoes along the curve. Its value is +1 iff the considered Jordan curve surrounds a vortex/sink/source. Such an index is computed around each pixel using a small closed curve. Around each vortex/sink/source, a small blob (whose size depends on the size of used curve) of +1 -index pixels is obtained. The number of blobs is $K$, and the center of the $i$ th blob provides $s_{i}$.

At this stage it remains to estimate radii $r_{i}$, and parameters $\left(a_{i} \cdots f_{i}\right)$ for each $i$. For a given radius $r_{i}$, the minimization of $H_{3}$ w.r.t. $\left(a_{i} \cdots f_{i}\right)$ is a non-linear leastsquares problem that can be readily solved using iteratively reweighted least-squares. Minimization of $H_{3}$ w.r.t. radius $r_{i}$ is more involved. Gradient descent techniques could be used to this end. Instead, we designed a simpler heuristic. It is reminiscent to the approach in [10] where one tries to find a compromise between the most robust linear regression (using a large neighborhood) and the less biased regression (not using a neighborhood larger that the actual "linearity" domain). Starting from a small value of $r_{i}$, we make it grow. For each new value of $r_{i}$, the associated parameters $\left(a_{i} \cdots f_{i}\right)$ are computed. If the corresponding singular point :

$$
\left[\begin{array}{l}
x_{c} \\
y_{c}
\end{array}\right]=\left[\begin{array}{l}
x_{i} \\
y_{i}
\end{array}\right]+\left[\begin{array}{cc}
c_{i} & d_{i} \\
e_{i} & f_{i}
\end{array}\right]^{-1}\left[\begin{array}{l}
a_{i} \\
b_{i}
\end{array}\right]
$$

remains within the concerned blob of +1 -index pixels, the estimation is considered as reliable, and we proceed. If not the procedure is stopped and the previous value of $r_{i}$ is selected. The $K$ parametric models defined through the $\theta_{i}$ 's interact via $\mathcal{H}_{3}$. Using a block Gauss-Seidel minimization 
we update them iteratively according to:

$$
\begin{gathered}
\theta_{i}=\left[\sum_{s \in D_{i}} \xi_{s}\left(P_{i}(s)^{T} P_{i}(s)\right)\right]^{-1} \sum_{s \in D_{i}} \xi_{s} P_{i}(s)^{T}\left(\boldsymbol{w}_{s}+\mathrm{d} \boldsymbol{w}_{s}-\right. \\
\left.\left[\boldsymbol{w}_{l a m}(s)+\sum_{k \neq i} P_{k}(s) \theta_{k}\right]\right)
\end{gathered}
$$

where $P_{i}(s) \triangleq\left[\begin{array}{cccccc}1 & 0 & x-x_{i} & y-y_{i} & 0 & 0 \\ 0 & 1 & 0 & 0 & x-x_{i} & y-y_{i}\end{array}\right]$, for $s=(x, y)$.

So far, laminar component $\boldsymbol{w}_{l a m}$, and dense field to be refined $\boldsymbol{w}$ were assumed as given. As usual in incremental multiresolution techniques, the latter one is provided, via simple interpolation, by the dense field finally estimated at previous resolution. As for the laminar component, it could simply be considered as a global translation to be estimated in some way [14]. We tried to be less restrictive. The laminar field is taken as the velocity field estimated at the coarsest resolution with the coarsest estimation grid of the multigrid technique proposed in [13]. This very smooth field is then properly interpolated on the current resolution.

\section{Experiments}

We report here experiments on the three different kinds of Meteosat images: the infrared channel, the visible channel and the water vapor channel (Fig. 2 and 3). In these experiments we used the same robust function in the three energy terms (namely $\rho(x)=1-\exp \left(\frac{x^{2}}{\sigma}\right)$ ). As for the parameter values we used the same ones in all the experiments (except for the visible image sequence where we imposed a higher regularization term). In the three cases the velocity fields seem physically plausible; they are also in accordance with what can be expected based on simple visual inspection of the sequences.

Let us notice the essential role played by the laminar flow. Indeed, in situations where there is a large global motion (such as in the water vapor sequence Fig. 2a), taking this "transport" field into consideration allows to recover accurately the location of depressions despite the displacement of their centers, and to extract secondary vortices which would be lost in the whole flow otherwise (see the vortex in the lower right corner of the water vapor image in Fig 2a).

The combination of the different flow representations provides very interesting results. The parametric field allows to get a physical interpretation of the flow. For example, the main swirls (corresponding to depressions), as well as counter-vortices are well captured. A sink and a source can also be seen in the right lower corner of the infrared

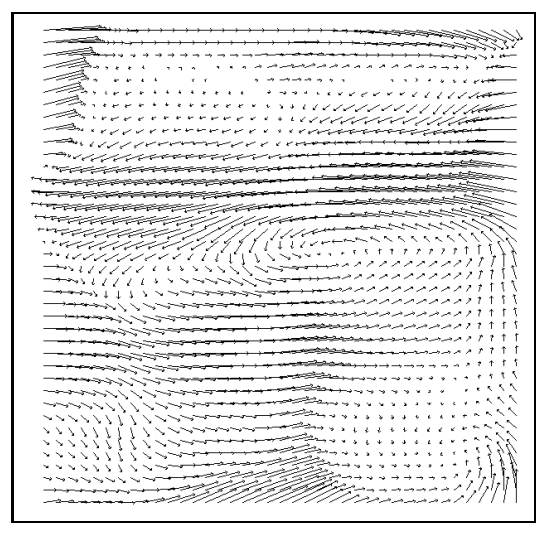

Figure 4. Flow computed with the generic model [13] on the visible image (similar set of parameter)

sequence (Fig. 3). Beside these structures of interest captured by the parametric field, complex and less structured information is carried by the laminar component and the estimated dense velocity field.

It is also interesting to point out the benefit of the parametric modeling in areas with low photometric gradient. Indeed, in these areas (as well as in regions with many data outliers) velocity fields estimated by the way of generic motion estimator (equ. 1) are poorly constrained and the nonlinear smoothness term tends to introduce inexistent discontinuities around and within these regions (see Fig. 4). By contrast, the parametric prior introduced here circumvents in an elegant way this problem: In such areas, the field is not only dependent on the spatial context, it is also guided toward a smooth solution having much more physical sense (see Fig. 2b).

\section{Conclusion}

In this paper we have proposed a method to estimate fluid flows. This method relies on the extension of a generic robust motion estimator. This extension consists in considering in a coupled way the estimation of the velocity field and of some structures of interest which are explanatory to the unknown flow. The method has been tested on the three kinds of Meteosat satellite images. The recovered flows seems to be in accordance with the underlying phenomena. Compared to classical (first-order regularization based) optical-flow estimators, this method allows a more robust estimation in large areas of low photometric contrast and a better extraction of highly diverging or swirling motions. Finally, the method provides simultaneously a dense estimation of the flow and a structured parametric representation which is believed to retain relevant physical information. 


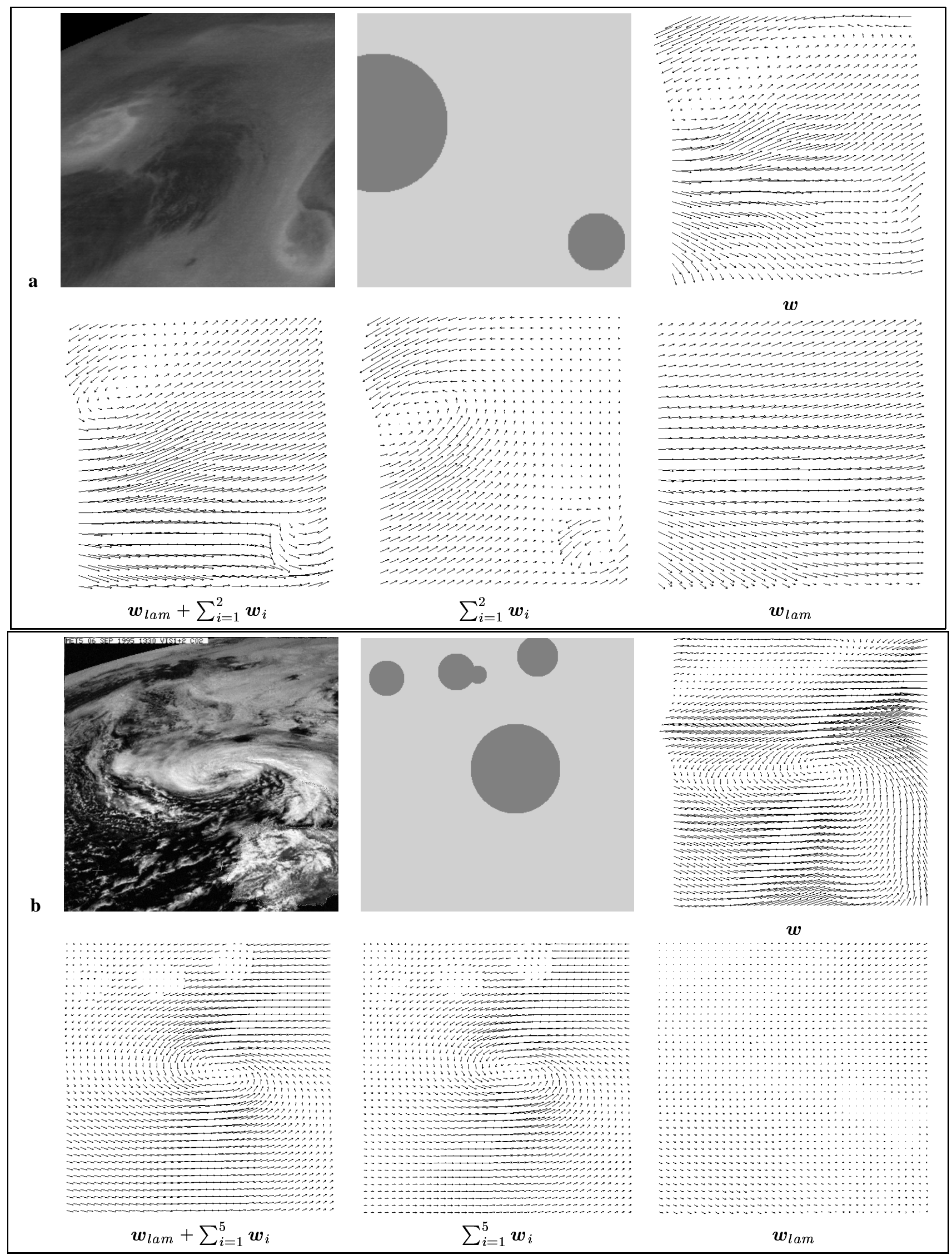

Figure 2. Result sample on satellite Meteosat images: (a) water vapor images and (b) visible images; extracted vortices/sinks/sources $\left(\left\{s_{i}, r_{i}\right\}_{i=1}^{K}\right)$, with $K=2$, resp. 5; estimated dense fields $\boldsymbol{w}$; estimated parametric and laminar fields $\boldsymbol{w}_{\text {lam }}+\sum_{i=1}^{K} \boldsymbol{w}_{i}$; parametric field alone $\sum_{i=1}^{K} \boldsymbol{w}_{i}$; laminar field alone $\boldsymbol{w}_{\text {lam }}$. 


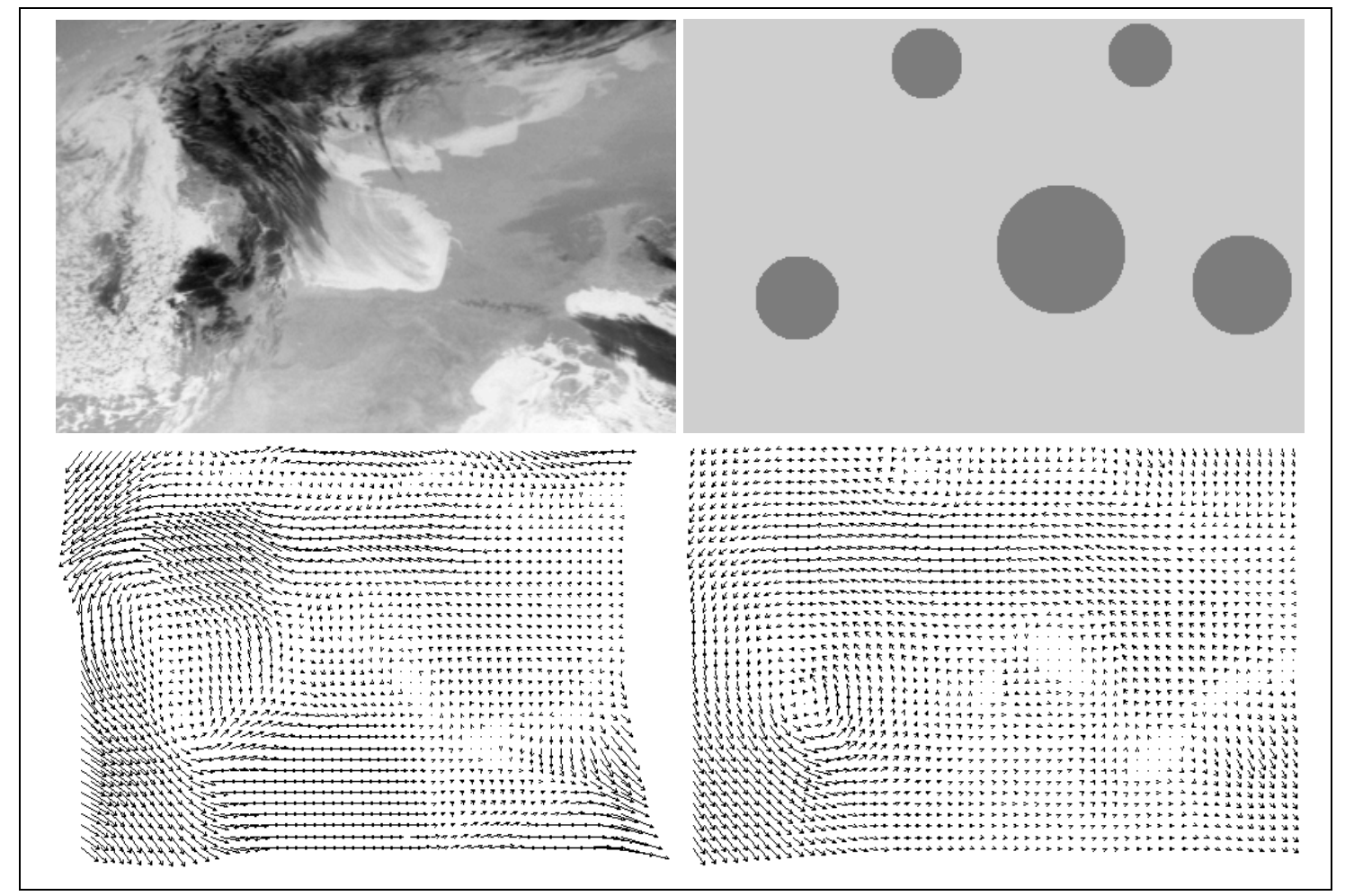

Figure 3. Results on infrared satellite Meteosat images; extracted vortices/sinks/sources $\left(\left\{s_{i}, r_{i}\right\}_{i=1}^{K}\right)$, with $K=5$; estimated dense fields $\boldsymbol{w}$; estimated parametric and laminar fields $\boldsymbol{w}_{l a m}+\sum_{i=1}^{K} \boldsymbol{w}_{i}$.

\section{References}

[1] J. Bergen, P. Anandan, K. Hanna, and R. Hingorani. Hierarchical model-based motion estimation. In $E C C V^{\prime} 92$, volume 558 of LNCS, pages 237-252. Springer-Verlag, 1992.

[2] M. Black and P. Anandan. The robust estimation of multiple motions: parametric and piecewise-smooth flow fields. CVIU, 63(1):75-104, 1996.

[3] M. Black and A. Rangarajan. On the unification of line processes, outlier rejection, and robust statistics with applications in early vision. IJCV, 19(1):75-104, 1996.

[4] P. Charbonnier, L. Blanc-Féraud, G. Aubert, and M. Barlaud. Deterministic edge-preserving regularization in computed imaging. IEEE Trans. Im.Proc., 6(2):298-311, 1997.

[5] I. Cohen and I. Herlin. Optical flow and phase portrait methods for environmental satellite image sequences. In ECCV’96, pages II:141-150, Cambridge, UK, Apr. 1996.

[6] W. Enkelmann. Investigation of multigrid algorithms for the estimation of optical flow fields in image sequences. CVGIP, 43:150-177, 1988.

[7] R. Ford and R. Strickland. Representing and visualizing fluid flow images and velocimetry data by nonlinear dynamical systems. Graph. Mod. Im. Proc., 57(6):462-482, 1995.

[8] D. Geman and G. Reynolds. Constrained restoration and the recovery of discontinuities. IEEE Trans. PAMI, 14(3):367383, 1992.
[9] R. Larsen, K. Conradsen, and B. Ersboll. Estimation of dense image flow fields in fluids. IEEE. Trans. Geo. and Remote Sensing, 36(1):256-264, 1998.

[10] M. Maurizot, P. Bouthemy, and B. Delyon. 2d fluid motion analysis from a single image. In CVPR, pages 184-189, Santa Barbara, June 1998.

[11] E. Mémin and P. Pérez. Dense estimation and object-based segmentation of the optical flow with robust techniques. IEEE Trans. Im. Proc., 7(5):703-719, 1998.

[12] E. Mémin and P. Pérez. Joint estimation-segmentation of optic flow. In ECCV'98, Freiburg, Germany, June 1998.

[13] E. Mémin and P. Pérez. A multigrid approach to hierarchical motion estimation. In ICCV'98, Bombay, India, Jan. 1998.

[14] H. Nogawa, Y. Nakajima, and Y. Sato. Acquisition of symbolic description from flow fields: a new approach based on a fluid model. IEEE Trans. PAMI, 19(1), 1997.

[15] J. Wallace and J. Foss. The measurement of vorticity in turbulent flows. Annu. Rev. Fluid Mech., 27:469-514, 1995.

[16] J. Zhong, T. Huang, and R. Adrian. Extracting 3D vortices in turbulent flow. IEEE Trans. PAMI, 20(2):193-199, 1998. 